> Une décision judiciaire de 2008 concernant les « enfants nés sans vie» souligne que «tout fœtus né sans vie, à la suite d'un accouchement, peut être inscrit sur les registres de décès de l'état civil ». Elle a révélé que la prise en charge des fœtus morts est devenue une question de société et de droits pour les couples. La reconnaissance conférée à «l'enfant » mort in utero entraîne certes une nécessité d'accompagnement des parents mais suscite aussi des interrogations pour les professionnels de santé. Cette évolution pose la question de la place à donner au fœtus mort sans tomber dans des positions idéologiques sur la reconnaissance du fœtus en tant que personne. Elle permet de s'interroger également sur les priorités de santé publique, à un moment où, si le deuil parental est reconnu, aucun registre épidémiologique national spécifique concernant la mort fœtale n'existe à ce jour en France. <

\section{Corps des foetus mort-nés}

\section{Nouvelles représentations et nouveaux droits}

Grégoire Moutel, Michèle Goussot-Souchet, Isabelle Plu, Marion Pierre, Thomas Leclercq, Jean-Christophe Coffin, Nathalie Duchange

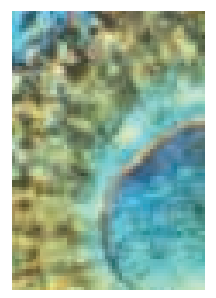

G. Moutel : laboratoire d'éthique médicale et de médecine légale, Université Paris Descartes, Faculté de médecine ; unité d'accès aux soins et droits des patients, Hôpital Corentin Celton, Assistance publique-Hôpitaux de Paris, France. gregoire.moutel@parisdescartes.fr

M. Goussot-Souchet : laboratoire d'éthique médicale et de médecine légale, Université Paris Descartes, Faculté de médecine ; maternité Cochin Port-Royal, Hôpital Cochin, Assistance publique-Hôpitaux de Paris, 123, boulevard Port-Royal, 75014 Paris, France.

M. Pierre : laboratoire d'éthique médicale et de médecine légale, Université Paris Descartes, Faculté de médecine ; département de médecine de l'enfant et de l'adolescent, Hôpital sud, CHU de Rennes,

16, boulevard de Bulgarie, BP 90347 , 35203 Rennes Cedex 2, France.

I. Plu, T. Leclercq, J.C. Coffin, N. Duchange:

laboratoire d'éthique médicale et de médecine légale, Université Paris Descartes, Faculté de médecine, 45, rue des Saints-Pères, 75006 Paris, France.

En droit, ce n'est qu'à la naissance, et à condition de naître vivant et viable, que l'enfant acquiert le statut de personne juridique. L'acte de naissance est réservé aux enfants nés vivants, même si le décès intervient dans les premières minutes qui suivent la naissance et que l'enfant est mort au moment de la déclaration, s'il est démontré qu'il a vécu. Un acte de naissance puis un acte de décès seront alors établis. Mais, en dehors de ce cas, se pose la question de la gestion du corps du fœtus mort soit spontanément in utero, soit dans le cadre d'une interruption de grossesse pour motif médical. Une décision de la Cour de cassation française du 6 février 2008 concernant les «enfants nés sans vie »a provoqué un débat public sur la question du devenir des fœetus issus de fausses couches ou d'interruptions médicales de grossesse. La haute juridiction a estimé en effet que, le code civil ne subordonnant l'établissement d'un acte d'enfant né sans vie ni au poids du fœtus, ni à la durée de la gestation, «tout fœtus né sans vie à la suite d'un accouchement peut être inscrit sur les registres de décès de l'état civil » [1]. Certains redoutent (bien que cela ne soit pas juridiquement fondé) que cette décision laisse à penser qu'à terme, par glissement des représentations, les fœtus soient assimilés à des personnes. Ainsi, il apparaît aujourd'hui nécessaire et essentiel de s'interroger sur la façon dont notre société peut permettre aux couples ayant perdu un fœtus de bénéficier d'une reconnaissance de cette perte et de pouvoir faire un travail de deuil. Il convient également de comprendre cette évolution en regard de nos valeurs collectives. Il s'agit alors d'analyser l'évolution de la gestion de la mort fœtale, en incluant, pour ceux qui le souhaitent, la question du rituel funéraire. Mais il faut veiller à ce que les réponses à ces demandes légitimes n'entraînent pas une remise en cause du statut des fœtus et ne rouvrent pas le débat sur la légitimité 
de l'interruption de grossesse. Cela n'est en effet ni la question initialement posée, ni la demande de la majorité des couples. De ce fait, il convient de clarifier les valeurs en jeu pour mieux informer les couples et organiser des prises en charge adéquates.

\section{Le fœtus : de la symbolique au concret}

Les fœtus nés sans vie se placent à la croisée des chemins entre les vivants et les morts. Cette place ambigüe explique que leur statut ait toujours posé question et leur non-existence en tant que personne juridique induit de nombreuses incertitudes.

Aristote, dans son Embryologie, expose la théorie du gradualisme : la semence masculine apporte la forme et le sang menstruel fournira la matière. L'opinion générale des Grecs dans l'Antiquité est qu'un fœtus symbolise l'idée d'enfant à venir. Pour Platon, dans le Dialogue sur la République, le terme «fétus» ne se rattache à aucune matérialité. Les Stoïciens, quant à eux, proposent de concevoir l'embryon comme une plante. Les religions monothéistes ne partagent pas ces conceptions: pour elles, l'embryon est le symbole de la vie divine et, au-delà de l'idée d'enfant, incarne une représentation sacrée. Mais, dans tous les cas, la perte fœtale est considérée comme un phénomène de régulation naturelle, sans conséquence et sans rituels.

Pour les Grecs dans l'Antiquité, à la naissance, le corps formé reçoit une âme immortelle et transcendante qui vient à sa rencontre, et pour les religions monothéistes, il reçoit l'esprit divin. Dans tous les cas, l'enfant n'est donc reconnu en tant que tel que lorsque son corps vivant est exposé au regard des autres membres de la cité. Avant cela, il n'a pas de concrétude.

Depuis les années 1960, l'avènement, d'une part, de la contraception qui permet une maîtrise de la procréation puis, d'autre part, de l'échographie obstétricale, fait porter un tout autre regard surle fœtus. Aujourd'hui, l'enfant est souvent le fruit d'un désir parental et devient, par ce fait, précieux dans l'imaginaire des parents. Actuellement, la première échographie est préconisée au premier trimestre de la grossesse. Ainsi, ce qui avait toujours été caché est mis en lumière avant même que la grossesse ne soit visible pour les tiers. Les parents rencontrent ainsi leur «bébé » pour la première fois à cette occasion et si le médecin ou la sage-femme ne trouve pas d'anomalie, l'examen devient convivial. L'échographie permet d'écouter les bruits du cœur, d'observer les mouvements des membres et la face, véritable «épiphanie du visage » qui, au sens de Levinas [2], inaugure la culture de la responsabilité et suscite un engagement.
Selon les psychanalystes $[3,4]$, le cadre échographique joue, au plan psychique, un rôle de catalyseur dans la personnification. L'image est le miroir de la parentalité et permet une incarnation du fœtus. Celuici devient concret, donc présent au monde avant sa naissance. Les images placent la pensée devant un contenu de significations affectives, sociales, médicales, mais aussi philosophiques, métaphysiques, anthropologiques. Ainsi les femmes et les couples s'attachent-ils à ce fœtus qui, pour eux, représente déjà leur enfant avec une forte «personnification symbolique» [3]. Cette évolution ne doit pas pour autant aboutir à l'assimilation du fœtus au statut de personne sur un plan légal. Une telle opinion relève des convictions et croyances individuelles, mais ne saurait être imposée à tous, d'autant qu'en filigrane elle remet en question l'interruption de grossesse. C'est pourquoi, pour sortir de ces éthiques de convictions, le Comité consultatif national d'éthique français soulignait, en 1984 [5], que l'embryon ou le fœtus doit être reconnu comme une personne humaine potentielle qui est ou a été vivante et dont le respect s'impose à tous.

Par ailleurs, la démarche de dépistage anténatal, lorsqu'elle révèle une anomalie, fait participer les couples à la décision de vie ou de mort. Dans la discussion sur une interruption de grossesse pour motif fœtal, les couples se retrouvent de facto face à une matérialité du fœtus puisqu'on leur montre des images, leur explique les anomalies corporelles du fœtus avec des schémas et leur parle du corps mort.

Ainsi, le fœtus devenu membre de la famille (avec la possibilité de clichés dans l'album de famille), mais aussi «patient » dans le cadre du diagnostic anténatal, devient aussi sujet d'une possible ritualisation du deuil.

La présence concrète du fœtus mort doit donc aujourd'hui être prise en compte puisque sa mort représente une réelle perte. Elle requiert dès lors, pour ceux qui le souhaitent et sans l'imposer à tous, une reconnaissance de ce deuil par l'entourage et une forme de ritualisation autour des corps. La situation est donc, aujourd'hui, très éloignée de celle qui prévalait au $\mathrm{XIX}^{\mathrm{e}}$ siècle et au début $\mathrm{du} \mathrm{xx}^{\mathrm{e}}$ siècle où ces corps étaient laissés aux institutions de soins et où les tissus embryonnaires et les fœtus étaient considérés soit comme «déchets anatomiques », soit comme objets de collections scientifiques.

\section{Une nécessaire clarification}

La question du devenir des fœtus morts dans notre société a été soulevée avec une certaine agitation médiatique lors du débat qui a fait suite à la découverte de corps fœtaux dans des maternités françaises en 2005 : des corps stockés qui n'avaient pas donné lieu à des rituels ni à une gestion commune avec les parents. Ce débat de 2005 a donc révélé à la société une procédure datant de 1993 [6] selon laquelle la ritualisation du deuil concernant le corps d'un fœtus mort n'était possible qu'au-delà des seuils de viabilité théorique proposés par l'Organisation mondiale de la santé (OMS) [7], soit à partir de vingtdeux semaines d'aménorrhée (SA) ou plus de 500 grammes de poids. En deçà de la limite théorique de viabilité, les fœtus étaient toujours considérés comme «déchets anatomiques». La question du rite funéraire était donc conditionnée au fait que, théoriquement, le fœtus 
aurait pu vivre. Cette situation, bien que n'ayant rien d'illégal, était porteuse d'une certaine violence pour les parents dont les fœtus étaient en deçà de ces seuils, et plus largement pour le grand public. Deux questions sont alors à explorer: quels sont le sens et la légitimité de ces seuils de viabilité et peut-on les appliquer à la ritualisation du corps fœtal mort?

Jusqu'en 1970, comme le souligne le rapport du comité d'experts de l'OMS de cette année [8], on admet traditionnellement que le fœtus est viable à la $28^{\mathrm{e}}$ semaine de gestation ou lorsqu'il pèse approximativement 1000 grammes. Mais cette définition apparaît, comme l'expose ce rapport, purement empirique, puisqu'elle repose sur l'expérience de cliniciens: on constate en pratique que les nouveau-nés d'un poids inférieur ont peu de chance de survivre alors que la mortalité décroît chez les nourrissons de plus de 1000 grammes. Le rapport souligne également que la classification internationale des maladies et des causes de décès de 1968 [9] entend par enfant vivant «l'expulsion ou l'extraction complète du corps de la mère, indépendamment de la durée de gestation, d'un produit de conception, qui après cette séparation, respire ou manifeste tout autre signe de vie, tel que battement du cœur, pulsation du cordon ombilical ou contraction d'un muscle soumis à l'action de la volonté ; tout produit d'une telle naissance est considéré comme vivant». Ce même rapport expose la crainte que les signes de vie ainsi mentionnés n'incitent à considérer comme vivants des fœtus manifestement trop jeunes et manifestement non viables et à considérer des enfants comme vivants y compris après interruption médicale de grossesse (où certains de ces signes peuvent être présents). La notion d'enfant né vivant n'est donc pas pour la médecine une définition suffisante et le groupe d'experts propose alors d'y adjoindre le concept de viabilité. Deux éléments sont liés à cette notion de viabilité : le poids de naissance et/ou le nombre de semaines de gestation. L'OMS propose alors que ces deux critères soient pris en compte pour définir dans les statistiques à venir la notion d'enfant né vivant et viable, et donc l'inscription à l'état civil, ainsi que dans les données de mortalité périnatale nationale et internationale, afin d'en faire la base des progrès à accomplir en néonatologie dans les différents pays. Ainsi liberté est donnée aux pays de «prendre en considération non seulement le poids, mais aussi la durée de la gestation calculée en jours à partir du premier jour de la dernière période menstruelle ». De manière étonnante, et toujours dans une approche empirique, le choix est fait de retenir pour la viabilité, d'une part le terme de plus de vingt semai- nes de gestation (22 SA) et/ou d'autre part, le poids de naissance supérieur à 500 grammes, alors que l'expérience et les témoignages de professionnels semblaient plaider pour vingt-huit semaines et 1000 grammes. Cette décision semble avoir été motivée par un désir de se donner de la «marge» mais on ne trouve aucun argument rationnel ou statistiquement valide pour attester de la légitimité de ces deux seuils finalement retenus. Les bornes de viabilité y apparaissent donc comme des notions incertaines. Ce sont pourtant ces dernières qui ont conditionné jusqu'à aujourd'hui la reconnaissance légale, administrative et symbolique de la viabilité.

La seconde étape fut celle qui, en France, en 1993, aboutit à statuer sur les fœtus qui auraient «droit» à une inscription sur le registre des décès, puis à des rites funéraires éventuels. Par automatisme et par simple analogie, sans qu'il y ait d'argumentaire éthique à ce choix, on énonça, par circulaire [6] - dont la valeur juridique est très faible - que seuls les fœtus au-delà des seuils de viabilité théorique rentreraient dans ce cadre. Cette décision présupposait qu'un enfant ne pouvait bénéficier d'une démarche administrative que dans le cas où, en théorie, il aurait pu vivre. Aucun débat public n'eut lieu sur ce sujet et la représentation nationale ne fut pas associée à ce choix.

C'est ainsi que, depuis 1993, seuls les fœtus de plus de 22 SA ou d'au moins 500 grammes, considérés comme viables, se voyaient appliquer le concept d' « enfants nés sans vie » pouvant bénéficier d'une inscription à l'état civil (en tant que fœtus décédés et non en tant que personnes). Ainsi, en pratique, la sage-femme ou le médecin établissait un certificat d'accouchement signalant «enfant né sans vie», ce qui permettait la dotation facultative d'un prénom et la possibilité d'inscription à la partie basse du livret de famille, après inscription sur le registre de décès de l'état civil. La prise en charge du corps était obligatoire, soit par la famille qui organisait des obsèques soit, à défaut, par l'institution (hôpital ou commune) qui procèdait à une crémation ou une inhumation.

Les fœtus mort-nés de moins de 22 SA et de moins de 500 grammes étaient simplement inscrits sur un registre hospitalier. Ils ne donnaient lieu en pratique à aucune mention sur les registres de l'état civil et le corps suivait souvent le même circuit de destruction que les pièces anatomiques.

La différence de prise en charge des corps en fonction du terme de la grossesse s'est avérée difficile à expliquer et à concevoir pour certains couples pour lesquels cette classification, issue d'un critère médical théorique sans rapport avec la situation vécue, ne correspondait pas à leur histoire et à leurs représentations. C'est ce que soulignent de nombreux travaux médicaux [10-12] ainsi que des travaux de nos collègues juristes pour qui la viabilité est une notion médicalement complexe et juridiquement infondée [13], tout comme ceux du Comité consultatif national d'éthique. Celui-ci expose clairement en 2005 [14] que « ks caractéristiques objectives et quantifiables (âge chronologique, poids, viabilité, pathologie, etc.) de la médecine et des lois risquent de heurter les représentations affectives que se font les parents de l'être en gestation et entraîner une souffrance et une demande de reconnaissance ». Ainsi, par exemple, pour un fœtus de vingt et une semaines, des parents sollicitant les mêmes droits à 
obsèques que pour un fœtus de vingt deux semaines se les voyaient refuser.

Au vu de ce débat, certaines communes ont mis en place au fil du temps des registres pour ces fœtus de moins de 22 SA appelés «registres des embryons », qui permettent l'organisation d'obsèques si les parents le souhaitent. Mais une telle pratique était rare, laissée à la discrétion des maires, et n'avait aucun caractère légal. De ce fait, on notait des inégalités sur le territoire national.

C'est donc la question de la légitimité d'une limite qui est posée. De la fécondation à la mort, la vie d'un être humain est une évolution continue passant par différents stades: stade embryonnaire, fœtus, nouveau-né, enfant, etc. Le passage d'un stade à l'autre se fait sans aucune discontinuité, et si pour la médecine il existe des états et des stades, tel n'est pas le cas pour les couples: il s'agit de leur enfant en devenir. Dans les pratiques, au sein des maternités, les équipes médicales qui gèrent ces situations constataient que, souvent, pour la femme et le couple, à quelques jours près, il s'agissait en fait d'une situation vécue de façon identique: celle d'une perte, d'une souffrance à laquelle il faut donner sens. II fallait donc répondre à des situations d'incompréhension, voire de tension. Aussi des parents se sont-ils adressés à la justice et les arrêts de la Cour de cassation de février 2008 ont été rédigés [1].

\section{Permettre une reconnaissance, sans remise en cause du statut de l'embryon ou du fœtus}

Cette jurisprudence a entraîné de nouveaux textes légaux en août 2008 qui font évoluer la réglementation existante [15-17]. Aucun terme seuil ni aucun poids de naissance n'est plus requis désormais pour l'inscription de l'enfant né sans vie sur les registres de décès à l'état civil. Cependant, pour éviter tout excès concernant des demandes où la morphologie de l'enfant expulsé ne correspondrait qu'à des amas cellulaires, et de manière à ne pas entraîner de confusion avec le domaine de l'interruption volontaire de grossesse, le modèle de certificat médical d'accouchement figurant en annexe de l'arrêté du 20 août 2008 rappelle que seul l'accouchement spontané ou provoqué pour raison médicale (interruption médicale de grossesse) peut donner lieu à l'établissement d'un tel certificat. En revanche, l'interruption spontanée précoce de grossesse (fausse couche précoce) et l'interruption volontaire de grossesse n'ouvrent pas droit à l'établissement de ce certificat. Cela revient à dire que l'acte d'enfant né sans vie ne peut être établi que s'il y a réellement un accouchement relevant de l'appréciation médicale des praticiens, c'est-à-dire impliquant le recueil d'un corps formé [18]. Cela veut dire de façon implicite que le certificat d'accouchement, et donc l'acte d'enfant sans vie, ne peuvent être établis qu'à partir de 14 SA, délai qui correspond, d'une part, au terme jusqu'auquel peut se pratiquer une interruption volontaire de grossesse pour détresse maternelle et, d'autre part, à la date limite de déclaration de la grossesse auprès des organismes sociaux.

Il faut bien noter que la reconnaissance de ces fœtus comme enfants nés sans vie ne leur confère en aucun cas une personnalité juridique. Ainsi, ils n'acquièrent pas le statut de personne, décision sage qui ne rouvre pas le débat sur l'interruption de grossesse (dépénalisée en France depuis 1975), qu'elle soit volontaire ou pour motif médical.

Cette reconnaissance répond à une situation vécue antérieurement par certains couples comme une forme de mépris et d'indifférence des institutions par rapport aux corps des fœtus, d'autant que leur prise en charge au sein de certains établissements ne répondait pas toujours à des critères de dignité et de transparence. Comme le souligne Axel Honneth dans ses travaux sur la reconnaissance [19], celle-ci doit savoir intégrer trois valeurs essentielles: I'amour, le respect des droits (au sens de règles collectives) et le respect de la dignité. On peut considérer que c'est ce qui est en jeu dans le cadre du questionnement sur le devenir des fœtus morts.

Il convient néanmoins de souligner ici que d'autres couples, en pareille situation, n'accordent pas les mêmes symboliques au fœtus ou ne souhaitent pas rentrer dans cette logique de la reconnaissance, qu'elle soit collective avec rite funéraire ou individuelle, tel par exemple l'habillage du corps fœtal, qui personnifie le fœtus comme l'enfant qu'il aurait dû être. C'est pourquoi il conviendra de ne pas imposer une même démarche à tous et de ne pas stigmatiser les choix.

\section{De nouvelles procédures légitimes mais oublieuses des impératifs épidémiologiques}

Ces récentes évolutions ont pour conséquence le fait que la déclaration, donc la traçabilité de la perte fœtale, peut dépendre uniquement de la volonté parentale. De plus, la procédure actuelle ne prévoit pas que les données sur les causes médicales du décès fœtal et sur les caractéristiques de la grossesse ne soient transmises à l'Institut national de la statistique et des études économiques (Insee) ou au Centre d'épidémiologie sur les causes médicales de décès de l'Inserm (CépiDc), comme cela est réalisé pour les décès survenant après la naissance. Pourtant la donnée de santé publique essentielle qu'est la mort fœtale devrait, elle aussi, être systématiquement colligée. Elle permet en effet, en analysant les causes des décès, de repérer de nouveaux facteurs de risque et donc un meilleur suivi des grossesses à venir par une amélioration des procédures et outils à mettre en œuvre en médecine périnatale. Avant la décision de justice de 2008, tous les décès de fœtus de plus de 22 SA ou de plus de 500 grammes faisaient l'objet d'une déclaration à l'état civil. II existait donc au moins une traçabilité en nombre, ce qui permettait une vision de santé publique au moins à partir de ce terme. 
La nouvelle situation laisse entrevoir plusieurs risques de confusion parmi lesquels le fait que tous les décès ne seront pas notés (ceci dépendant du choix des parents), y compris pour les plus de 22 SA ou de 500 grammes. On se trouve donc de facto devant une situation qui, si elle privilégie les droits et les choix des couples, ne prend pas en compte l'intérêt collectif en termes scientifiques. Les modifications réalisées ne permettent pas d'organiser, par le biais de l'état civil, le recueil des informations nécessaires à l'établissement des indicateurs de mortinatalité (rapport du nombre d'enfants sans vie à l'ensemble des enfants nés vivants et des enfants nés sans vie) et de mortalité périnatale (rapport du nombre d'enfants sans vie et d'enfants décédés à moins de 7 jours à l'ensemble des enfants nés vivants et des enfants nés sans vie).

La solution existe et pourrait voir le jour : il s'agirait de dissocier deux démarches. D'une part, celle des couples qui souhaitent ou non ritualiser la perte fœtale à travers une inscription à l'état civil, d'autre part, la création d'un registre des morts fœtales où seraient systématiquement inscrites (quitte à envisager les nécessaires démarches d'anonymisation) les données essentielles aux travaux de santé publique et de recherche biomédicale, à savoir : le terme de la grossesse au moment de la perte fœtale, les caractéristiques de la grossesse (simple ou multiple, spontanée ou issue d'une assistance médicale à la procréation), l'étiologie de la mort fœtale in utero ou diagnostic ayant conduit à une interruption médicale de grossesse ou à une interruption sélective de grossesse [20].

On constate donc que le débat s'est à ce jour focalisé, certes de manière légitime, sur la demande parentale, mais que d'autres éléments doivent aujourd'hui faire évoluer la réflexion pour prendre en compte, au-delà de l'intérêt individuel, l'intérêt collectif. Les professionnels se sont mobilisés en ce sens pour conserver un outil épidémiologique et une réflexion sur ce sujet est en cours au ministère de la Santé.

\section{Conclusion : éviter des dérives pernicieuses}

La question du devenir des morts, quels qu'ils soient, est au cœur de toutes les visions anthropologiques des civilisations. «Qu'est devenu son corps? » est donc une question essentielle et légitime pour des parents. Des représentations plus matérialistes et plus biomédicales des fœtus ne doivent pas occulter la réalité de leur personnification par certains couples. Ces différences d'approche doivent pouvoir s'intégrer à l'accompagnement psychologique, au travail de deuil et à la prise en charge de la mort fœtale dans les institutions en intégrant les dimensions spirituelles éventuelles. Pour autant, une telle démarche ne doit pas être imposée à tous, certains ne souhaitant pas conférer une telle charge émotionnelle à la mort fœtale. Ainsi, le grand défi pour les soignants, les institutions de soins et les professionnels du monde funéraire sera de savoir informer et proposer, sans pour autant imposer ou décider à la place des couples.

II s'agit donc, face au fœtus mort, d'avoir une attitude de responsabilité prenant en compte les évolutions des demandes sociales en termes de reconnaissance de la souffrance liée à la perte d'un fœtus. Cette demande doit être respectée pour ce qu'elle est, en prenant garde pour autant de ne pas personnifier à outrance le fœtus [21]. Une personnification pourrait en effet, le cas échéant, être à l'origine de deux dérives: une psychopathologie de la perte fœtale plaçant le fœtus au même niveau qu'un être cher dont on aurait partagé la vie (un ami, un parent ou un enfant né vivant) et/ou une remise en cause du statut juridique de l'embryon ou du fœtus visant à lui donner le statut de personne, ce qui remettrait en cause le délicat équilibre aujourd'hui instauré sur cette question dans notre société. Les récentes évolutions semblent avoir évité ces deux écueils. $\diamond$

\section{SUMMARY}

\section{Fetuses born lifeless:}

\section{new representations and new rights}

A february-2008 rule about «children born lifeless» stressed that « every fetus born lifeless » could be registered at the General Register Office. This rule, which was followed in 2009 by the implementation of new procedures to deal with dead fetuses, highlighted that this topic is more and more becoming an important public issue. The rights for parents to choose has now been recognized, bringing about new questions for health professionals. Another point is which symbolism is to be given to dead fetuses to alleviate couples' suffering, without being too ideological, i.e. seeing fetuses as persons. This change also makes professionals reconsider public health system priorities, and this at a very particular time. Indeed, as parent mourning has now been made easier through registration at the General Register Office, there has not been any rule yet linking national epidemiological registration and fetal death in France. $\diamond$

\section{CONFLIT D'INTÉRÊTS}

Les auteurs déclarent n'avoir aucun conflit d'intérêts concernant les données publiées dans cet article.

\section{RÉFÉRENCES}

1. Cour de cassation, première chambre civile. Arrêt $n^{\circ} 128$ du 6 février 2008, France, www.courdecassation.fr/jurisprudence

2. Levinas $\varepsilon$. Totalité et infini. Norwell : Kluwer Academic Publishers, 1981.

3. Mitchell LM. Baby's first picture. Ultrasound and the politics of fetal subjects. Toronto : University of Toronto Press, 2001 : 200-48 (258 p).

4. Missonnier S, Golse B, Soulé M. La grossesse, l'enfant virtuel et la parentalité. Éléments de psychopathologie périnatale. Paris: Presses Universitaires de France, 2004.

5. Comité consultatif national d'éthique. Avis $n^{\circ} 1$ sur les prélèvements de tissus d'embryons et de fœtus humains morts, à des fins thérapeutiques, diagnostiques et scientifiques, 1984, www.ccne-ethique.fr

6. Ministère de la Santé. Circulaire du 30 novembre 2001 relative à l'enregistrement à l'état civil et à la prise en charge des corps des enfants décédés avant la déclaration de naissance, citant Circulaire du 22 juillet 1993 relative à la déclaration des nouveau-nés décédés à l'état civil et circulaire conjointe des ministères de la Solidarité, de la Justice et de l'Intérieur en date du 30 novembre 2001, France. 
7. Pignotti MS. The definition of human viability: a historical perspective. Acta Paediatr $2010 ; 99$ : 33-6.

8. Organisation mondiale de la santé. Rapport technique $n^{\circ} 457$, prévention de mortalité et de la morbidité périnatales. Genève : 0MS, 1970.

9. Organisation mondiale de la santé. Manuel de la Classification internationale des maladies, traumatismes et causes de décès. Genève : OMS, 1968.

10. Wolff JR, Nielson PE, Schiller P. The emotional reaction to a stillbirth. Am J Obstet Gynecol 1970 ; $108: 73-7$.

11. Lake MF, Johnson TM, Murphy J, Knuppel RA. Evaluation of perinatal grief support team. Am J Obstet 1987 ; $157: 1203-6$.

12. Dumoulin M, Blondel B, Lequien P. Naître et ne pas être. J Gynecol Obstet Biol Reprod $1992 ; 22: 385-92$.

13. Philippe C. La viabilité de l'enfant nouveau-né. Recueil Dalloz Sirey $1996 ; 4: 29-32$

14. Comité consultatif national d'éthique. Avis $n^{\circ} 89$ à propos de la conservation des corps des fœtus et enfants mort-nés. Réponse à la saisine du premier ministre, 2005, www.ccne-ethique.fr

15. Décret du 20 août 2008 relatif à l'application du second alinéa de l'article 79-1 du code civil, France.
16. Arrêté du 20 août 2008 relatif au modèle de certificat médical d'accouchement en vue d'une demande d'établissement d'un acte d'enfant sans vie. France.

17. Décret du 20 août 2008 modifiant le décret $n^{\circ} 74-449$ du 15 mai 1974 relatif au livret de famille. France.

18. Circulaire interministérielle du 19 juin 2009 relative à l'enregistrement à l'état civil des enfants décédés avant la déclaration de naissance et de ceux pouvant donner lieu à un acte d'enfant sans vie, à la délivrance du livret de famille, à la prise en charge des corps des enfants décédés, des enfants sans vie et des fœtus. France.

19. Honneth A. La lutte pour la reconnaissance. Traduction de P. Rusch. Paris : Éditions du Cerf, 2000 (édition originale: 1992).

20. Goussot-Souchet M. Interruption sélective de grossesse pour pathologie grave d'un jumeau : étude des pratiques auprès des Centres pluridisciplinaires de diagnostic prénatal. Université Paris Descartes : mémoire de master de recherche en éthique, 2005, www.ethique.inserm.fr

21. Gellman-Patucca S, Dugué $C$, Belghiti $\varepsilon$, et al. The risk of drift in the management of perinatal loss. Concerning the 20 of august 2008 new legislation allowing registration of all fetuses. J Gynecol Obstet Biol Reprod 2008 ; 37 : 815-6.

\section{TIRÉS À PART}

G. Moutel

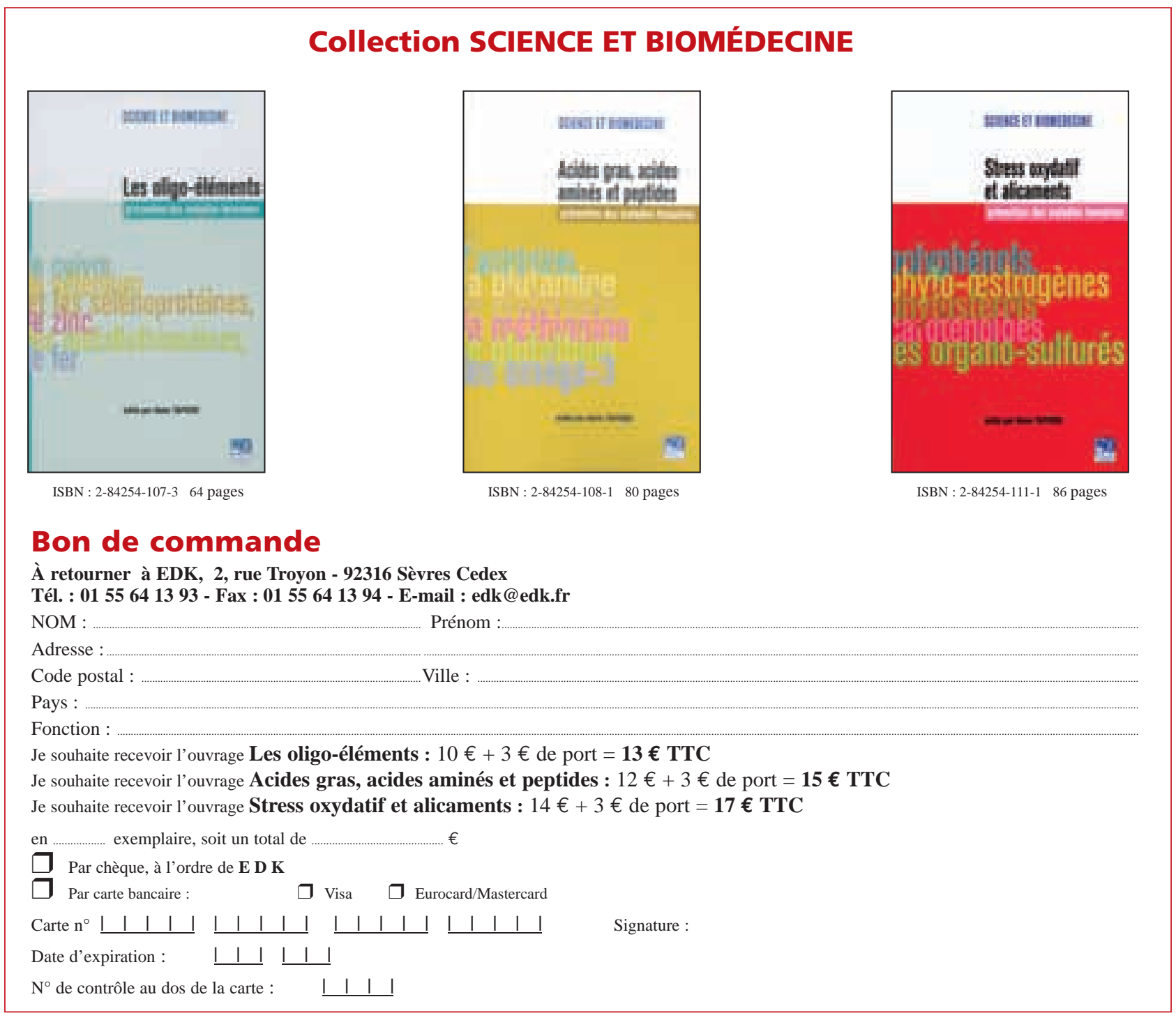


Médecine/Sciences est une revue internationale mensuelle francophone d'information dans tous les domaines de la biologie et de la médecine.

Les articles sont référencés dans PubMed depuis 2003, à l'exception des Brèves.

$\varepsilon n$ écrivant dans $\mathrm{m} / \mathrm{s}$, les auteurs ont le plaisir de faire partager aux lecteurs, dans leur langue, leur intérêt et même leur enthousiasme pour le sujet traité, en y apportant leur touche d'humour et de culture, surtout dans la partie Forum, où la plus grande liberté d'expression est autorisée.

Toutefois, la revue s'adressant à un lectorat très varié de scientifiques confirmés, d'enseignants, d'étudiants et de médecins, elle exige de la part des auteurs de développer leurs sujets, en allant jusqu'au bout des connaissances scientifiques, quelle qu'en soit la difficulté. Cela est particulièrement vrai pour les Synthèses, états de l'art sur un sujet donné, dans un style clair et intelligible pour les non-spécialistes.

La rédaction de la revue demande à tout auteur de se conformer aux quelques règles de base suivantes : $\bullet$ toujours définir les sigles et acronymes; - rassembler en tableaux, glossaires, les précisions techniques, méthodologiques et les compléments d'information qui surchargeraient le texte.

\section{Les 3 rubriques de $\mathrm{m} / \mathrm{s}$}

1. Des Synthèses qui font le point sur un sujet par un (des) auteur(s) spécialiste(s) du domaine. Au-delà du catalogue des faits collectés, elles doivent permettre une vraie discussion des résultats scientifiques. Des Dossiers techniques (exposé d'une technique ou d'un ensemble de techniques récemment développées dans le domaine biomédical).

2. Le Forum propose des articles de réflexion, c'est-à-dire, des Perspectives et des Chroniques sur des sujets faisant l'objet de débats dans la communauté scientifique, ainsi que des revues sur l'histoire biomédicale, les sciences sociales et la santé, la santé et l'environnement... Les Faits et Chiffres traitent de sujets dans les champs de l'épidémiologie, de la démographie, de l'économie de la santé..., avec des données chiffrées sous forme de tableaux.

3. Le Magazine est le reflet de l'actualité scientifique, faisant état, dans des textes courts, de résultats originaux importants récemment publiés. II est constitué de Nouvelles, spontanées ou sollicitées, et de Brèves, courtes notes de lecture.

\section{Normes générales de présentation} des articles

Tous les articles doivent être soumis par voie électronique via le système Fontisworks (http://msc.fontismedia.com). La procédure est indiquée sur le site.

Attention : veiller à ce que les courriels provenant du site de gestion fontismedia ne soient pas reconnus comme spams par votre serveur et éliminés. Autoriser l'adresse msc. fontismedia.com

Tous les articles doivent être accompagnés des coordonnées de tous les auteurs : • nom et prénom, - institution, - adresse professionnelle, téléphone, télécopie, courriel. Les Synthèses et les textes de la partie Forum ne peuvent excéder 18000 caractères (espaces compris, références exclues), 30 références et 3 à 5 illustrations (figures et tableaux), avec un titre en français et en anglais. Ils doivent être accompagnés d'un résumé d'environ 700 caractères destiné à offrir un aperçu rapide du sujet, et d'un résumé en anglais d'environ 1000 caractères, qui apparaîtra dans PubMed.

Il est demandé aux auteurs de mentionner les articles parus dans $\mathrm{m} / \mathrm{s}$ sur le sujet traité dans les dernières années (rechercher dans Pubmed).

\section{Les Nouvelles}

6000 à 8000 caractères au maximum (espaces compris, références exclues), 10 références au plus et 1 à 2 figures. Titre en français et en anglais.

\section{Les Brèves}

2000 caractères au maximum (espaces compris), maximum de 5 références ne mentionnant que le premier auteur et les références du journal. $\varepsilon x$ : Dupont T et al. Science $2010 ; 352: 458-9$.

Textes et tableaux en fichiers Word (enregistrements en .doc, format PDF exclus) illustrations en fichiers séparés. Tableaux et illustrations appelés dans le texte. Les références citées dans les tableaux doivent apparaître sous forme de $\left[\mathrm{n}^{\circ}\right]$ et être incluses dans la liste des références.

Illustrations: schémas en format Illustrator ou PowerPoint, photos en format jpeg ou tif. Lorsque nécessaire, l'échelle de l'image doit figurer dans l'illustration et sa valeur indiquée dans la légende. Légendes complètes et détaillées des figures et tableaux intégrées en fin de texte. Illustrations en chiffres arabes (ex: Figure 1) et tableaux en chiffres romains (ex: Tableau II). Les illustrations doivent faire l'objet d'une demande d'autorisation de reproduction, si nécessaire.

\section{Présentation des références}

Appelées dans le texte par leurs numéros entre crochets ([1], [2], [3-5]) et classées par ordre d'apparition dans l'article.

Mentionner tous les noms des auteurs, suivis des initiales de leurs prénoms, jusqu'au nombre de 4 . Au-delà, les 3 premiers le sont, suivis de et al. (en italique).

Le style $M / S$ est inclus dans la liste des styles référencés dans EndNote (voir liste complémentaire « get more on the web » téléchargeable à partir de la rubrique style manager du site Endnote)

\section{Pour les articles de revues scientifiques}

Exemple: Sivori S, Falco M, Della Chiesa M, et al. CpG and double-stranded RNA trigger human NK cells by Toll-like receptors : induction of cytokine release and cytotoxicity against tumors and dendritic cells. Proc Natl Acad Sci USA 2004 ; 101 : 10116-21.

\section{Pour les ouvrages}

Exemple : Kupiec JJ, Sonigo P. Ni Dieu ni gène. Paris : Seuil, $2004: 230$ p.

\section{Pour les chapitres d'ouvrages}

Exemple : Ménard D, Beaulieu JF, Boudreau F, et al. Gastrointestinal tract. In : Unsicker K, Krieglstein K, eds. Cell signaling and growth factors. New York: Wiley, 2005 : 755-90.

Très important: les auteurs sont priés de mentionner tout conflit d'intérêt potentiel concernant le manuscrit soumis à publication dans $\mathrm{m} / \mathrm{s}$, en particulier de nature financière. Cette information sera gardée confidentielle par la rédaction de $\mathrm{m} / \mathrm{s}$ jusqu'à la publication de l'article.

\section{Rédaction Paris}

secretariat@medecinesciences.org tél : 0155641393

Rédaction Québec : medecine.sciences@bellnet.ca 